\title{
Robotic Etching: The creation of digital etching using robotics
}

\author{
Verity Winslow \\ Centre for Fine Print \\ Research, University of \\ the West of England, \\ Bristol, BS3 2JT, UK \\ Verity.Lewis@uwe.ac.uk
}

\author{
Paul O'Dowd \\ Faculty of Engineering, \\ University of Bristol \\ Bristol, BS81UB, UK \\ paul.odowd@bristol.ac.uk
}

\author{
Ian Chamberlain \\ Centre for Fine Print \\ Research, University of \\ the West of England, \\ Bristol, BS3 2JT, UK \\ lan.Chamberlain@uwe.ac.uk
}

\author{
Carinna Parraman \\ Centre for Fine Print Research, \\ University of \\ the West of England, \\ Bristol, BS3 2JT, UK \\ Carinna.Parraman@uwe.ac.uk
}

\begin{abstract}
This paper looks at the potentials afforded by robots when applied to traditional printing techniques such as etching. Through experiments using an adapted drawing robot, this study explores the possibilities of creating works where a traditional etching needle is placed into a robot, opening up a new field of digitally enabled etching. This work is conducted through a case study with the artist and printmaker lan Chamberlain.
\end{abstract}

Etching. Robotics. Digital. Technology. Printmaking. Drawing. Letterpress.

\section{INTRODUCTION}

This paper describes an investigation of a robot used within the traditional etching printmaking process. The use of a robot has been studied in two ways. First, for the novel affordances an autonomous robot provides within printmaking, and secondly, as a study of how robotic technologies can be integrated within a traditional process. In this second aspect, the first author has worked as mediator, facilitator and researcher between the robot as tool and with lan Chamberlain as artist. This paper therefore reflects on both the accessibility and utility of robotics within art as a new tool, and upon the potential expressive capabilities of a robot within the etching printmaking field.

Technological advances, particularly within the field of robotics in recent years, have led to new possibilities when it comes to producing artwork. New robotic machines capable of both creating and physically rendering artefacts have opened new areas of investigation around machine creativity and human/robotic collaboration.

This paper looks at the potentials afforded by robots when applied to traditional printing techniques such as etching. Through experiments using an adapted 3D printing robot, this study explores the possibilities of creating works where a traditional etching needle is used by a robot.
Printmaking is a process-led method of image making and reproduction, and printmakers use a range of specialist tools and equipment (Laidler 2011). The traditional etching medium has a reliance on physical mark making, which is captured on the print plate. Purely digital approaches, i.e. predominantly screen based digital media, do not sufficiently emulate the nuances of tactile interaction.

William Ivins, Keeper of Prints at The Metropolitan Museum New York, in 1953, described prints as pictorial statements, each demonstrating a particular syntax, where each process demonstrates a definite look or identity. Whilst syntax is generally used in linguistics to describe the grammatical structure of a sentence, in this context and likewise Ivin's understanding, pictorial syntax refers to the relationship of the artist's gesture, the mark made by the tool, and the printing process to create a reproducible image. Of course, Ivins in the mid $20^{\text {th }}$ century was writing on the impact of photographic technologies, could not have foreseen the impact of digital technologies for the artist. Mac Holbert, Master Printer of Nash Editions explains how artists in the 80s were confronted with new tools and processes that, like photography a century and a half earlier, created great controversy (White 2006). With the emergence of digital technologies, these traditional qualities were considered as a threat to the fine qualities of the printmaker's mark resulting in a digital ubiquity and homogeneity. 
Today, we know that this is not the case. There is a potential to explore the opportunity to transform purely digital information through the physical mechanisms of a robot. Robotic drawing has demonstrated some capability for nuanced mark making, however, it is not clear how accessible this is, how well it can be used, how valuable it is or how effective it is in complement to human expression. This paper is an initial study into this area.

\section{HISTORY OF ETCHING}

Traditional etching was established in the middle ages and involves a metal plate covered with a wax ground that is resistant to acid. The artist then scratches into the ground with a pointed etching needle where they desire a line to appear in the finished piece, so exposing the bare metal. The plate is immersed in acid, which bites into the exposed lines and areas. When ink is applied, these incised marks hold a residue of ink. The surface is wiped clean, damp paper is placed on top of the plate, and heavy pressure is applied through a rolling press.

Due to the tangible nature of drawing onto a plate, etching, up to this point, has been difficult to combine with digital means. Unlike screen-printing that utilises digitally printed transparent film, or digitally prepared flexo plates for letterpress, etching still relies on the physicality of the hand drawn line. Robotic technology provides a new alternative. Via robotic technology, digital imagery can be translated into robotic movement and gesture. This application of robotics into etching opens up new possibilities for automated markmaking and drawing within the domain of the etch print process.

\section{ROBOTIC DRAWING MACHINES}

Robots that can emulate the physical mark-making process have been evident in history for many years. Some of the first examples were produced as early as the $18^{\text {th }}$ century.

Pierre Jaquet-Droz's 'The Draftsman', and 'The Writer', designed in the late 1770 s, could be considered as two of the first drawing and handwriting machines. Both automatons imitated a boy sitting at a table with the ability to draw small portraits or write short sentences. Both the images and text images could be interchanged by adapting the machine's mechanics. These devices contained over 6,000 moving parts, could achieve incredibly refined results. Even the pressure on the quill could be adjusted. Many believe that the automata built at this time were the world's first programmable robots (Herath \& Kroos 2016).
More recently there have been a variety of interesting projects utilising robotics to create artworks.

Patrick Tresset, a Lecturer of Creative Robotics and Prof. Frederic Fol Leymarie, a programmer, have developed a series of robots (mainly called Paul) with either one or two robotic drawing arms. Using a webcam, Paul takes an image of his sitter, or still life, and then executes the algorithmic and robotic procedures needed to produce the drawing (Tresset \& Fol Leymarie 2013).

MEART, described as the semi-living artist, is collaboration between the University of Western Australia, Georgia Institute of Technology and the Ultrafuturo Group. The project developed a pneumatically actuated robotic arm to create drawings, as controlled by a living network of neurons from rat cortex grown on a multi-electrode array (MEA). The model system consisted of living neurons, growing in the laboratory for Neuroengineering at Georgia Tech, and connected by Internet to the pen-wielding metal and plastic pair of arms behaving in gallery exhibitions around the world (Bakkum et al. 2007)

Since 2009, The University of Konstanz in Germany have been developing eDavid, a painting machine that mimics humans distributing real paint onto canvas. Using a visual feedback loop, it evaluates its results, correcting errors and adapting its painting approach accordingly (Deussen, Lindemeier, Pirk \& Tautzenberger 2012).

Portuguese artist Leonel Moura, has developed Artsbot employing swarm robotics technology. Utilising multiple mobile robots, Artsbot creates abstract images produced from emergent behaviour derived from the robots interactions (Moura 2007).

There are several other projects that could be mentioned here but where we feel our research differs from many of these contemporary art-robotic investigations is that our primary focus is to examine if a robot can be combined with a traditional practice to allow a new digital outcome to be realised.

\section{CASE STUDY}

To investigate new ways that printmakers could exploit digital technologies and robotics to adapt and develop traditional etching methods, we conducted a case study with artist lan Chamberlain, who has built his creative expertise in drawing and etching. Due to the exacting nature offered by both digital software and robotic hardware, the research also looks to consider if 
new techniques could be realised that were previously problematic or impossible to obtain through traditional practices.

Digital drawing devices and tablets have allowed artworks to be realised in a wealth of different ways. Tools within vector software permit artists and designers to create imagery with near perfect precision. The ability to delete and instantly reinstate elements of a composition permits a freedom not obtained through conventional drawing methods.

Likewise, the ability to digitally recreate, rotate, overlay and rescale objects also opens up new possibilities that were previously challenging or extremely time-consuming using traditional approaches.

As previously stated, digital methods have been widely embraced by many contemporary printmaking practitioners, where etching has been one of the last to be integrated into the digital realm. Through this case study we Through this case study we make an initial study of how new robotic technology might be adopted by artists as part of the etching discipline. To achieve this, the first author becomes a mediator, facilitator and researcher between the robot and the artist. Through this framework we hope to:

- Study a contemporary artist's working techniques.

- Gain a better understanding of why artists might be interested in using this technology.

- Explore the way in which they wish to use the technology as a component of their practice.

- Investigate how the technology may inform artist's traditional working methods.

- Obtain an outsider's perspective on the development of the machines.

- Investigate drawing techniques.

\section{INTRODUCTION TO THE ARTIST}

Ian Chamberlain is an artist and printmaker predominately working within the field of etching. His work is held in internationally renowned collections including the V\&A and the Tate Modern. He has been shortlisted for both the Jerwood and John Ruskin Drawing Prize. His work takes reference from an on-going interest in manmade technological forms of industrial structures such as bridges, the scientific radio telescopes at Goonhilly Satellite Earth Station, and ex-military structures such as the Acoustic sound mirrors.

The majority of the subjects and locations he records were once considered at the forefront of technology during their lifetime. Some of those technologies are now defunct or have been reconfigured for different uses. The subject matter therefore is echoed in the process used to record it. The etching process enables him to make a sustained enquiry into the subject's structure, location and the effects of time passing. It becomes his own visual experience and a graphic equivalent to an observed moment in time.

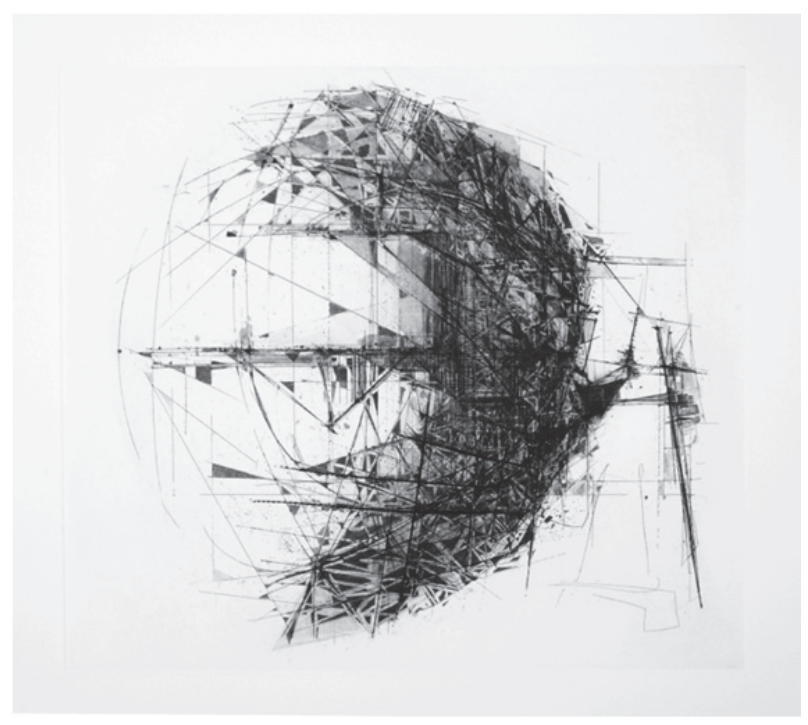

Figure 1: Sat.VI, 2015 @ Ian Chamberlain.

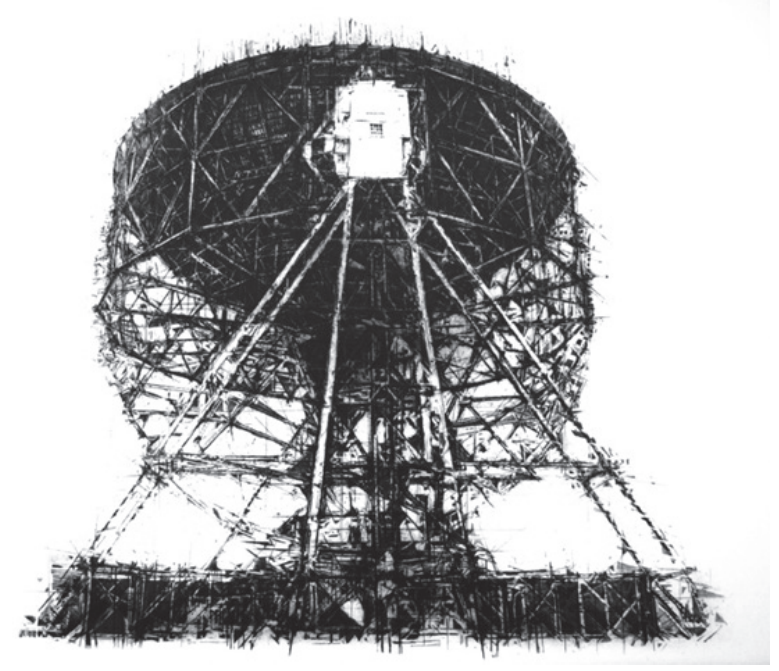

Figure 2: Transmission V, 2018 @ lan Chamberlain.

\section{WHY WAS THE ARTIST SELECTED?}

Chamberlain's work was selected for a number of reasons, predominantly due to its complexity and high level of detail. In striving to recreate such intricacy, via a robotic implementation, it would enable us to gain a better understanding of the robot's capability and potential limitations.

Another interesting aspect of Chamberlain's work lies within its combination of accuracy and delicacy. Although the images are very precise, to a level 
that could be compared to that of a machine, they have a distinct hand drawn quality that separates them.

Through exploring the reproduction of theses images with a robot we hope to gain insight on human gesture and whether it is something that can be emulated or complemented by a machine. Chamberlain's subject matter and play on technology also seemed to be very fitting for this investigation.

\section{TESTING CONDUCTED}

The first step was to conduct a basic feasibility study to ascertain if one of Chamberlain's drawings could be reproduced by the robot. To do this, a drawing provided by the artist was digitalised and initially drawn by the robot using a pen to gain a clear indication of how the robot was translating it.

The image was then manipulated in vector software to determine a more refined result. This assessment involved analysing the robotic drawing, comparing it to the original sketch. Although the onscreen image appeared to be homogeneous to the original, it was only when the image was output through the robot that we could ascertain a better understanding of which aspects needed to be adjusted. Due to the digitalisation of the image, this on-going manipulation was easily achieved, removing and adding areas of shade where desired. After a series of iterations, a test image was agreed.

The ability to develop and adapt the image before the plate is etched is a crucial change in the engraving process. To remove lines in traditional etching you have to use burnishing techniques, which can be very time consuming. Through the robot's capability of first drawing the image on paper, the artist can reduce the amount of corrections needed when transcribing the image to the copper plate.

It is also within this iterative process that we start to examine human gesture. By comparing the robots marks to that of Chamberlain's pencil lines we can start to analyse the way the artist has intuitively built up the composition and creation of depth and shade. As well as this giving us a better understanding of the tacit and easily overlooked ways in which humans draw. By a process of comparison to the artist's original work, it is also possible to examine the potential for subtly and nuances' within the robot's own mark-making process.

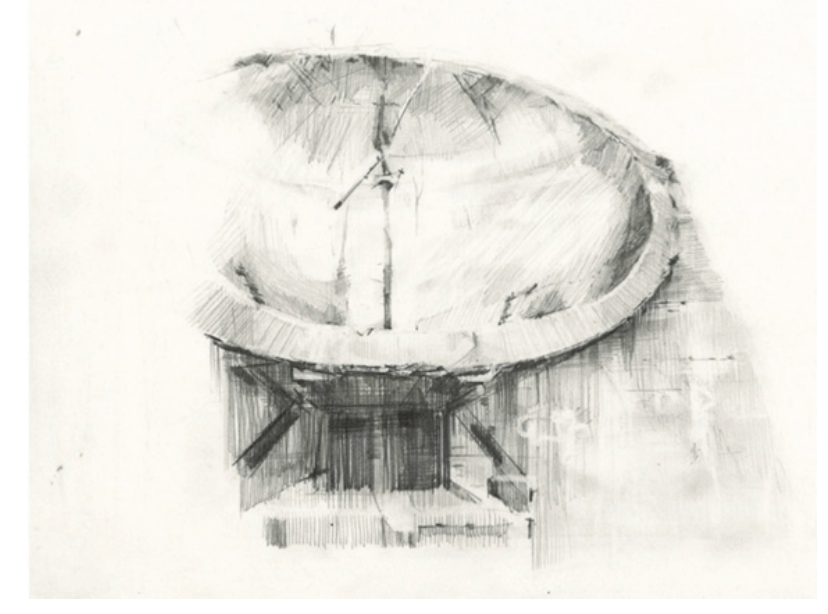

Figure 3: Original pencil drawing provided by the artist.

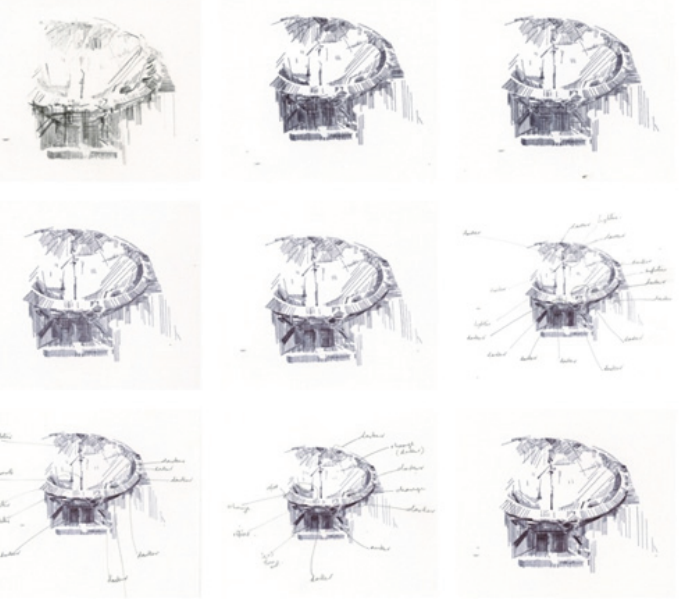

Figure 4: Drawing robot iterations.

After the refinement of the digital drawing, validated by using the robot as a pen-plotter (pen and paper), the robot was set to work on the copper plate customary to etching. A copper plate was prepared with hard ground in the traditional way and an etching needed was inserted into the robot, rather than a pen. The plate was then added to the drawing surface of the robot, and scribed upon.

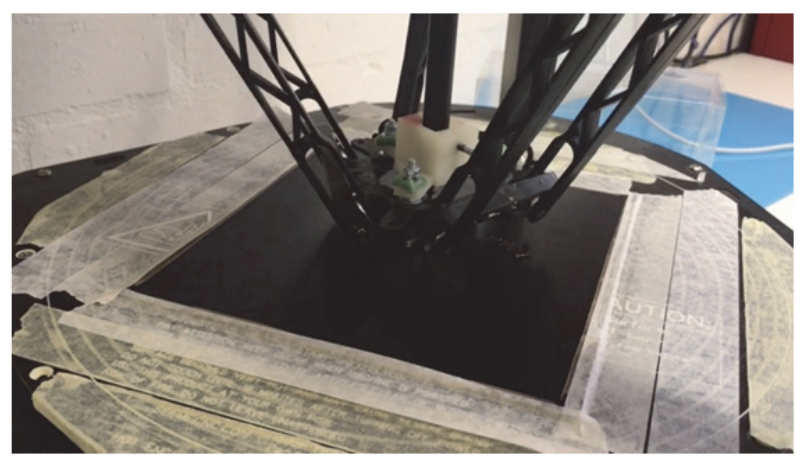

Figure 5: Robot etching the plate. 
We found that the robot was proficient at drawing into the ground and the image was successfully scribed into copper. The scribed plate was then processed and printed in the traditional way, resulting in the image in Figure 6.

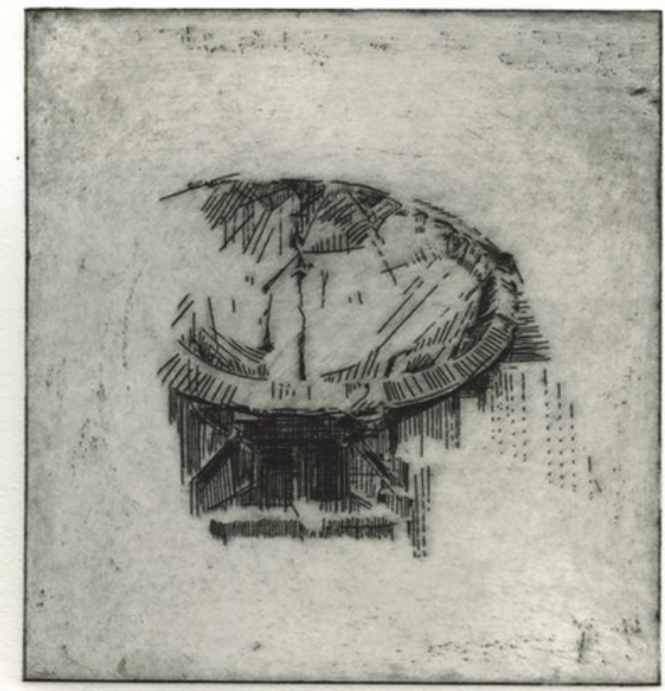

Figure 6: First test etching.

lan's final prints are made up of many phases, which include much reworking of the plate. He also incorporates techniques such as aquatint, stopping out and burnishing until the desired outcome is achieved. As such, the results from the robotic process at this early stage cannot be fully compared to lan's finished etchings. However, as an initial starting point, there is clearly interesting potential.

To explore our preliminary mark-making findings further, another of Chamberlain's images was digitalised, this time both drypoint and hard ground etching were investigated to see if the machine was able to engrave into a variety of materials and how the printed outcomes varied. This would enable us to determine the most suitable substrate and tools for the robotic process.

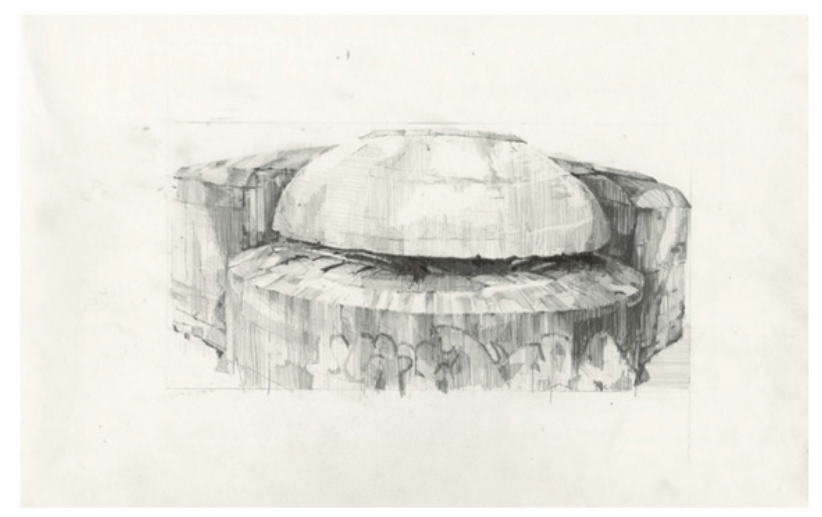

Figure 7: New pencil drawing provided by artist.
The robot struggled at times with the drypoint due to the pressure needed to engrave the material. This was observable by the motors not achieving their target positions. The drypoint printing method, where no acid bath is used, also meant that it was much more difficult to acquire a variation in printed line depth and therefore hard ground etching was considered more complementary.

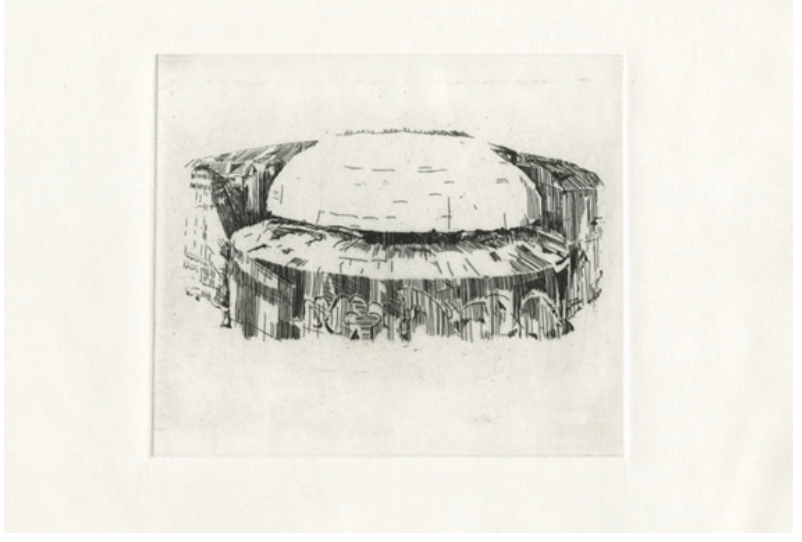

Figure 8: Hard ground etching.

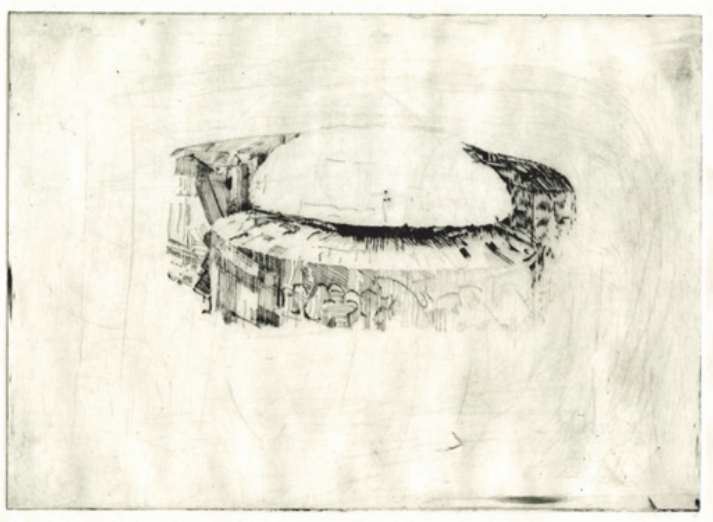

Figure 9: Drypoint etching.

\section{RESULTSI ANALYSIS}

The results were then fed back to the artist and a dialogue opened up to discuss the potentials of the medium. The first observation was that as a starting point, from nothing to the given results, the outcomes are surprisingly positive; the robot was well suited to the method.

It was agreed that the most obvious and realistic uses of the robot during the early stages of development could be to speed up the process. Chamberlain's etchings can take months to achieve and being able to realise the composition digitally could save time. It was also felt that the robot could be utilised to produce under-drawings, which the artist could then rework. The ability to explore 
pattern and repeat elements were also of interest, as was the opportunity to experiment with scale

In regards to further areas of development, the robots ability to produce a dot matrix could lend itself well to the Mezzotint printmaking method, also part of the intaglio family. This could also be used to create halftone, something that is considered very difficult using the conventional hand-rendered means. Experimenting with miss-registration, overlapping and tiling could produce some interesting variation and was considered as an area that needed further investigation. Digital typography and vector graphics could also bring a new aesthetic to the medium, which could open up etchings appeal to a wider audience.

Potential problems were also highlighted. The artist felt that roughly only about forty per cent of his etching process lies in the line generation. To obtain the real elegance and depth associated with many fine works, supplementary practices would have to be introduced. Further research would be needed to see if the robot was able to contribute to aspects such as burnishing and/or aquatint, or whether these would remain as post-processes to a robot enabled etching process.

Another concern that was raised resided around the removal of the human touch. For many, a large part of etching's charm lies within its reliance on the haptic and the craftsmanship with the handrendered technique. However, as with many other traditional methods, the introduction of digital aspects may change how some artists approach the medium but it is unlikely to cause the traditional method to disappear. Rather, robot enabled etching may provide a new alternative process for artists to explore.

\section{CONCLUSION}

With an increasing number of artists utilising more digital means and tablets as a drawing method, it makes sense to look at new ways to output these images. A robot with its ability to make marks in a physical manner analogous to that of human gesture lends itself particularly well for this use. At this time, the robotics is mainly located within research labs and industry. However, as robotic technology becomes more widely available, the potentials for its application within art are clear.
As seen with letterpress in more recent years, a new generation of tech savvy artists are revitalising a traditional method through digital means. Historically within printmaking, with each new technological breakthrough there has been a desire to rekindle traditional processes by combining it with cutting edge technology. Finding ways to translate digital representation into gesture through robotics may newly afford graphic elements and typography, which not generally associated the etching field

As with all new technological developments within the art field, it generates fears of traditional techniques being striped of their unique haptic quality. In contrast, our hope is to add something new, and to reinvigorate interest within the print medium. Due to the fundamental differences between people and machines, necessary robotsolutions will always need to be co-developed, and will provide interesting disparities in both technique and effect for the purpose of etching. Hopefully with these new opportunities and challenges, a new breed of digital etcher will arise.

\section{REFERENCES}

Bakkum, D. J. et al. (2007) MEART: The semiliving artist, Frontiers in Neurorobotics, 1. doi: 10.3389/neuro.12.005.2007

Deussen, O., Lindemeier, T., Pirk, S. and Tautzenberger, M. (2012) Feedback-guided stroke placement for a painting machine, pp.25-33.

Herath, D. and C. Kroos. (2016) Robots and Art: Exploring an Unlikely Symbiosis. Springer.

Ivings, W. M. (1953) Prints and Visual Communication. MIT Press, Cambridge

Laidler, P. (2011) Collaborative digital and wide format printing: Methods and considerations for the artist and master printer. $\mathrm{PhD}$, University of the West of England.

Moura, L. (2007) A new kind of art: The robotic action painter. $X$ Generative Art Conferece, Politecnico di Milano University.

White, G. (ed.) (2006) Nash Editions: Photography and the Art of Digital Printing, New Riders.

Tresset, P. and Fol Leymarie, F. (2013) Portrait drawing by Paul the robot. Computers \& Graphics. 37, pp.348-363. 